improve access to our service and indicates a potential path for digital transformation within respiratory outpatient physiotherapy.

\section{P69 DOES PATIENTS' EXPERIENCE IMPROVE ON THE PREM- 9 AFTER A 6-WEEK PULMONARY REHABILITATION PROGRAMME?}

SM Chavaillaz, EJ Chaplin, E Dayes, S Ward, SJ Singh, D Coope, L Houchen-Wolloff. Centre for Exercise and Rehabilitation Science, Pulmonary Rehabilitation Department, Leicester, UK

\subsection{6/thorax-2021-BTSabstracts.179}

Introduction Evidence suggests that PR has a huge impact on a number of outcomes for patients with Chronic Obstructive Pulmonary Disease (COPD). ${ }^{1}$ However, limited data exists that has measured patients' experience of COPD pre and post rehabilitation. This study aimed to quantify the impact of PR on the experience of COPD patients using the Patient Reported Experience Measure 9 (PREM-9). ${ }^{2}$ The PREM-9 is a questionnaire designed to help health care professionals (HCPs) learn more about the patient experience of living with COPD.

Methods Patients with COPD attending a 6 week PR programme in the United Kingdom consented to use their clinical data, including the PREM-9.

SPSS was used to analyse the change in PREM-9 total score, and individual items post PR. The change in PREM-9 was correlated with the change in other routinely collected data COPD Assessment Test (CAT), Hospital Anxiety and Depression Scale (HADS) and the Incremental Shuttle Walk Test (ISWT).

Results Data from 69 participants was analysed, 35 males, mean [SD] $\mathrm{FEV}_{1}$ (\%) 57.3\% [19.6], age 66 [10.2] years. A significant mean change was noted for the PREM-9 of -3.13 (95\%CI: -5.39 to -0.88 ). Change in individual PREM-9 questions and domains are reported in table 1.

There was a weak positive correlation between the PREM9 questionnaire change with the change in HADS anxiety domain $(r=0.35, p=0.04)$ and the change in CAT questionnaire $(r=0.38, p=0.02)$. However, no significant correlation

\begin{tabular}{lllll} 
Abstract P69 Table 1 & COPD PREM-9 results & & \\
\hline & & $\begin{array}{l}\text { Pre PR } \\
\text { Mean } \pm[S D]\end{array}$ & $\begin{array}{l}\text { Post PR } \\
\text { Mean } \pm[S D]\end{array}$ & P value \\
& & & \\
\hline PREM-9 & Question 1 & $2.79 \pm 1.5$ & $2.05 \pm 1.49$ & \\
My everyday life & Question 2 & $1.4 \pm 1.53$ & $1.5 \pm 1.25$ & \\
with COPD & Question 3 & $2.28 \pm 1.44$ & $1.72 \pm 1.2$ & \\
& Question 4 & $1.79 \pm 1.44$ & $1.37 \pm 1.27$ & \\
PREM-9 & Question 5 & $2.14 \pm 1.55$ & $1.84 \pm 1.27$ & \\
Usual care in COPD & Question 6 & $1.45 \pm 1.33$ & $1.17 \pm 1.01$ & \\
& Question 7 & $1.64 \pm 1.3$ & $1.02 \pm 0.87$ & \\
PREM-9 & Question 8 & $1.93 \pm 1.32$ & $1.67 \pm 1.44$ & \\
COPD exacerbation & Question 9 & $1.88 \pm 1.38$ & $1.7 \pm 1.39$ & \\
PREM-9 & Total score & $17.19 \pm 9.26$ & $14.05 \pm 7.76$ & $.008^{*}$ \\
HAD Anxiety & Total score & $8.54 \pm 5.43$ & $6.49 \pm 4.74$ & $.005^{*}$ \\
HAD Depression & Total score & $6.72 \pm 3.94$ & $5.7 \pm 4.09$ & .098 \\
CAT & Total score & $22.79 \pm 7.7$ & $19.43 \pm 6.31$ & $.003^{*}$ \\
ISWT & Total score & $291.71 \pm 149.33$ & $338.29 \pm 158.9$ & $.000^{*}$ \\
\hline * statistical difference $P=<0.05$ & & & \\
& & & &
\end{tabular}

was found between the PREM-9 change and the HADS depression domain or ISWT change.

Conclusion Patients' experience of living with COPD improved after a 6-week PR programme especially in coping with their COPD every day. These changes were associated with positive changes in anxiety and symptoms. Therefore, this questionnaire provides a unique insight into the patients' experience with their care and may act as a quality indicator for benchmarking the PR service.

Ethics Number: 17/EM/0156

\section{REFERENCES}

1. Spruit, et al. An official American Thoracic Society/European Respiratory Society statement: key concepts and advances in pulmonary rehabilitation. AJRCCM 2013;188(8):e13-e64.

2. Hodson., et al. Development and first validation of a patient-reported experience measure in chronic obstructive pulmonary disease (PREM-C9). Thorax 2019;74 (6):600-603.

\section{P70 THE IMPACT OF POST COVID-19 REHABILITATION ON HOSPITAL AND NON-HOSPITALISED PARTICIPANT- IS THERE A DIFFERENCE?}

${ }^{1} \mathrm{SJ}$ Singh, ${ }^{2} \mathrm{AC}$ Watt, ${ }^{2} \mathrm{~L}$ Houchen-Wolloff, ${ }^{2} \mathrm{~S}$ Ward, ${ }^{2} \mathrm{EJ}$ Chaplin, ${ }^{2} \mathrm{NY}$ Gardiner, ${ }^{2} \mathrm{E}$ Daynes, ${ }^{1}$ University of Leicester, Leicester, UK; ${ }^{2}$ University Hospitals of Leicester NHS Trust, Leicester, UK

\subsection{6/thorax-2021-BTSabstracts. 180}

Background There is evidence that those hospitalised with COVID-19 have a significant and persistent symptom burden. Early data suggests that symptoms and functional deficit may be favourably influenced by a structured rehabilitation programme. There is little data describing the impact of a rehabilitation programme on those who had COVID-19 but were not hospitalised and managed in the community who also have persistent burdensome symptoms. We therefore wanted to explore early outcome data from rehabilitation post infection and specifically explore data from those referred from the community to our rehabilitation programme.

Methods Participants were recruited to a 6 week out-patient supervised rehabilitation programme, with sessions twice a week. The programme was developed specifically for the post COVID-19 population using educational materials from the YourCovidRecovery website. The outcomes were: the incremental shuttle walking test (ISWT) (including a familiarisation test at baseline), the endurance shuttle walking test (ESWT), COPD Assessment Test (CAT), Functional Assessment of Chronic Illness Therapy Fatigue Scale (FACIT), Hospital Anxiety and Depression Scale (HADS), EuroQual 5 domains (EQ5D) thermometer, the Montreal Cognitive Assessment (MoCA) and quadriceps maximum voluntary contraction (QMVC) (chair based strain gauge). Data was analysed using SPSS v25. A paired/independent t-test was used to compare changes before and after rehabilitation between and within groups.

Results $\mathrm{N}=82$ individuals completed the COVID-19 rehabilitation programme (45 male, mean [SD] age 58.1(16.2) years), 49 white British. 62 individuals were admitted to hospital (11.8(14.8) days) and 20 non-hospitalised. Overall the data identified significant improvements in the whole group for the ISWT, ESWT HADS (D), CAT,FACIT, QMVC 


\section{Abstract P70 Table 1}

All

$$
\begin{aligned}
& \text { Hospitalised } \\
& \text { Mean } \\
& \text { difference (SD }
\end{aligned}
$$

Non-

Hospitalised

difference

(SD)

\begin{tabular}{lllll}
\hline ISWT(m) & $100.1(95.6)^{* *}$ & $95.5(90.0)^{* *}$ & $114.4(112.8)^{* *}$ & $18.9(70.7,-32.9)$ \\
ESWT(secs) & $332.0(636.9)^{* *}$ & $308.0(686.4)^{* *}$ & $417.2(422.2)^{* *}$ & $109.1(470.0,-251.7)$ \\
QMVC (kg) & $5.2(4.8)^{* *}$ & $5.6(4.7)^{* *}$ & $3.7(5.5)$ & $1.9(2.2,-6.0)$ \\
FACIT & $3.8(7.8)^{* *}$ & $3.4(7.4)^{*}$ & $5.1(9.2)^{*}$ & $1.8(-2.6,6.2)$ \\
CAT & $2.6(6.0)^{* *}$ & $2.8(5.6)^{* *}$ & $0.7(7.2)$ & $2.1(5.4,-1.1)$ \\
EQ-5D & $7.7(20.1)^{* *}$ & $11.0(19.4)^{* *}$ & $3.9(19.0)$ & $14.9(3.2,26.6)^{*}$ \\
(thermometer) & & & & \\
MoCA & $0.8(3.8)$ & $0.8(4.2)$ & $0.7(1.9)$ & $0.08(2.2,-2.4)$ \\
HAD-A & $0.6(3.1)$ & $0.6(3.0)$ & $0.4(3.3)$ & $0.2(1.5,-2.0)$ \\
HAD-D & $1.1(3.5)^{*}$ & $1.3(3.4)^{*}$ & $0.5(3.8)$ & $0.8(1.2,-2.7)$ \\
\hline${ }^{*}<0.05 * *<0.01$ & & &
\end{tabular}

and EQ-5D thermometer $(\mathrm{p}<0.05)$. With the exception of the EQ-5D there was no significant difference in improvements between groups $(\mathrm{P}>0.05)$ (table 1$)$. The within group changes demonstrated similar improvements in exercise capacity and the FACIT $(p<0.01)$ but the non-hospital group did not reach statistical significance for other outcomes.

Conclusion Early data suggests that those who have prolonged and significant symptoms post COVID-19 improve after a supervised rehabilitation programme. The response to the intervention is similar in both hospitalised and non-hospitalised groups. This is early cohort data and therefore must be treated with caution, nevertheless is encouraging.

\section{P71 PATIENTS WITH LONG COVID BENEFIT FROM REHABILITATION INDEPENDENTLY OF THE SEVERITY OF THE ACUTE COURSE OF THE DISEASE}

PS Schueller, JF Frommhold. MEDIAN Clinics, Berlin, Germany

\subsection{6/thorax-2021-BTSabstracts. 181}

Background Large numbers of people suffer from symptoms that continue or develop after acute COVID-19 like reduced exercise performance, impaired lung function and psychological distress. The need of post-corona rehabilitation to reduce long-term complications is becoming a key component in the continuum of care. We measured functional outcomes of Long Covid patients in two German rehabilitation centres.

Methods Measurements at admission and discharge of the inpatient rehabilitation stay (mean duration of stay: 27) comprised 6-minute walk distance (6MWD), vital capacity (VC), PHQ-9 and GAD-7. Compared were 3 groups: 1) acute course hospitalization in 2020 (hospitalized 2020); 2) acute course hospitalization in 2021 (hospitalized 2021); 3) without hospitalization (outpatient 2021).

Results In all groups the 6MWD increased significantly during rehabilitation in the pre to post comparison with an average of $+30.5( \pm 86.7)$ meters (outpatients 2021), $+42.5( \pm 99.9)$ meters (hospitalized 2021) and $+67.8( \pm 122.7)$ meters (hospitalized 2020). At discharge, patients in all groups improved in $\mathrm{VC}+150.3( \pm 350.2) \mathrm{ml}$ (outpatients 2021), +157.4 ( \pm 576.8$)$ $\mathrm{ml}$ (hospitalized 2021) and $111.3( \pm 377.3) \mathrm{ml}$ (hospitalized 2020). PHQ-9 decreased significantly in the pre to post comparison with an average of $-3.1( \pm 3.9)$ (outpatients 2021), $2.8( \pm 4.1)$ (hospitalized 2021) and $-3.2( \pm 4.0)$ (hospitalized $2020)$ as well as the GAD-7 -3.3 ( \pm 3.8$)$ (outpatients 2021), $3.0( \pm 3.9)$ (hospitalized 2021) and $3.7( \pm 4.2)$ (hospitalized 2020).

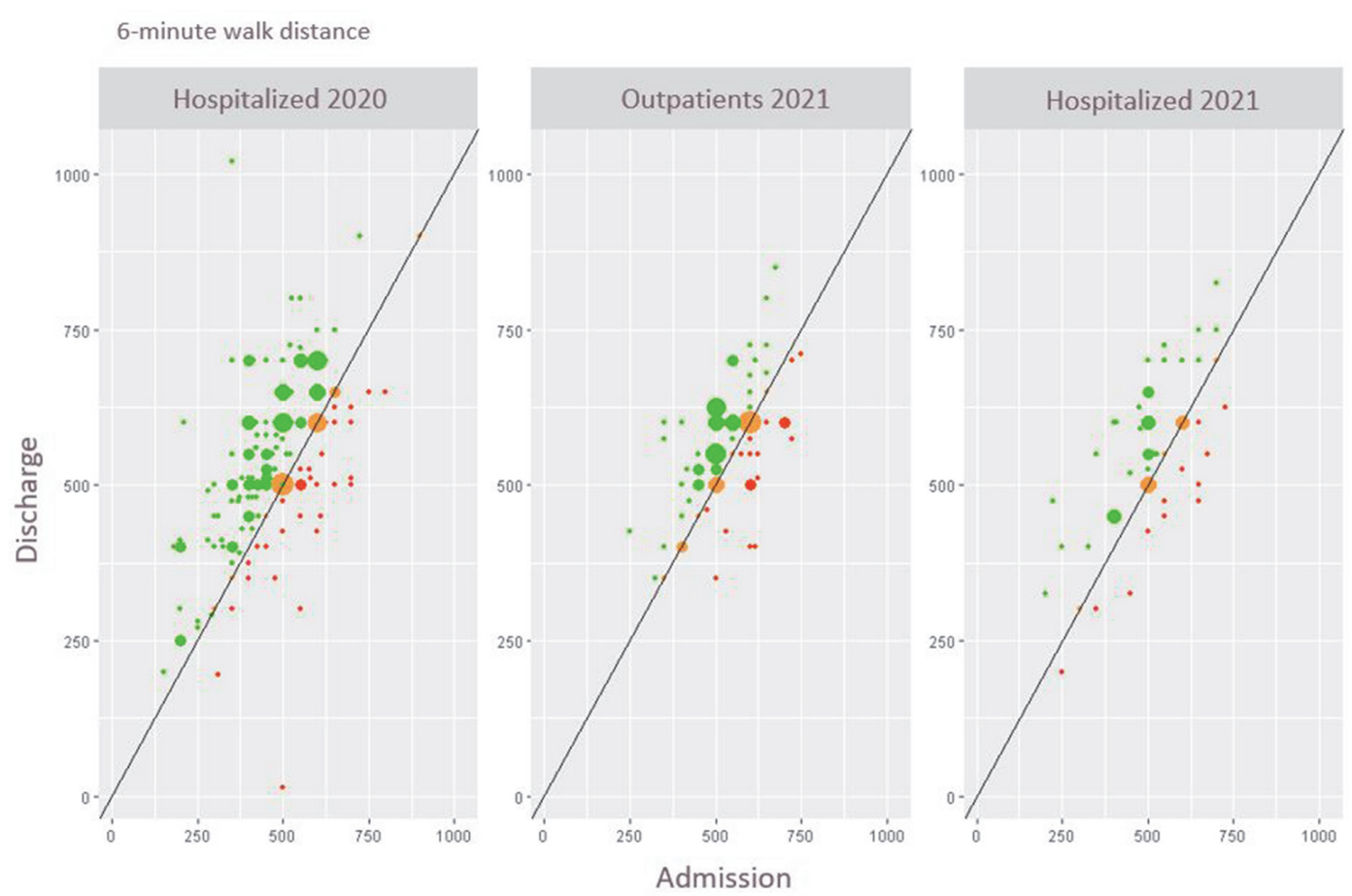

Abstract P71 Figure 1 